\title{
Fake Finger Detection by Finger Color Change Analysis
}

\author{
Wei-Yun Yau ${ }^{1}$, Hoang-Thanh Tran $^{2}$, Eam-Khwang Teoh ${ }^{2}$, and Jian-Gang Wang ${ }^{1}$ \\ ${ }^{1}$ Institute for Infocomm Research, 21 Heng Mui Keng Terrace, Singapore \\ ${ }^{2}$ Nanyang Technological University, EEE, Singapore \\ wyyau@i2r.a-star.edu.sg, tranhoangthanh@pmail.ntu.edu.sg, \\ eekteoh@ntu.edu.sg, jgwang@i2r.a-star.edu.sg
}

\begin{abstract}
The reliability of a fingerprint recognition system would be seriously impacted if the fingerprint scanner can be spoofed by a fake finger. Therefore, fake finger detection is necessary. This work introduces a new approach to detect fake finger based on the property of color change exhibited by a real live finger when the finger touches a hard surface. The force exhibited when the finger presses the hard surface changes the blood perfusion which resulted in a whiter color appearance compared to a normal uncompressed region. A method to detect and quantify such color change is proposed and used to differentiate a real finger from the fakes. The proposed approach is privacy friendly, fast and does not require special action from the user or with prior training. The preliminary experimental results indicate that the proposed approach is promising in detecting the fake finger made using gelatin which is particularly hard to detect.
\end{abstract}

Keywords: Fake finger, Color change, blood perfusion.

\section{Introduction}

The identity of an individual is a very critical "asset" of that individual that facilitates the person when performing myriad daily activities such as financial transactions, access to places and buildings and to computerized accounts. However, the traditional means of identity authentication using tokens such as card and key or personal identification number and password is becoming vulnerable to identity thefts. Therefore, the ability to correctly authenticate an individual using biometrics is becoming important. Unfortunately, the biometric system is also not fool-proof. It is subjected to various threats including attack at the communication channels (such as replay attacks), the software modules (such as replacing the matching module), the database of the enrolled users and the sensor with fakes [1], [2].

Recently, several researchers have shown that it is possible to spoof the fingerprint recognition system with fake fingers [3],[4]. These include enhancing the latent prints on the finger scanner with pressure and/or background materials to creating fingerprint molds using materials such as silicon, gelatin and Play-Doh as well as the use of cadaver fingers [5]. In order to counter such spoof attacks, fingerprint recognition 
system vendors have considered several approaches to detect the liveness of the finger. In general, these approaches can be classified into the following three categories [6]:

1. Analysis of skin details in the acquired images: minute details of the fingerprint images are used, ex: detecting sweat pores [5] and coarseness of the skin texture. A high resolution sensor is usually needed. Furthermore, sweat pores and skin texture varies with finger type, such as those dry and wet fingers and thus these approaches usually have large false error.

2. Analysis of static properties of the finger: additional hardware is used to capture information such as temperature, impedance or other electrical measurements, odor, and spectroscopy [12] where multiple wavelengths are exposed and the spectrum of the reflected light is analyzed to determine the liveness of the finger. Except for the spectroscopy technique, the other approaches can be easily defeated. However, the spectroscopy technique requires the use of expensive sensing mechanism.

3. Analysis of dynamic properties of the finger: analyzes the properties such as pulse oximetry, blood pulsation, perspiration, skin elasticity and distortion [6]. The former two approaches can only detect a dead or an entire fake finger but cannot differentiate false layer attached to the finger. In addition, it may reveal the medical condition of the user. To measure the perspiration, the user has to place the finger on the sensor for quite some time and may not be feasible for people with dry finger. A summary of the various liveness detection approaches is given in [7],[8].

Most of the approaches except the spectroscopy and the skin distortion techniques are not able to detect fake finger layer made using gelatin as gelatin contains moisture and has property such as electrical property quite similar to human skin. However, the spectroscopy technique requires expensive equipment while measuring the skin distortion requires the user to touch and twist the finger which is not user-friendly and will require user training. In this paper, the mold made using gelatin will be investigated as this is the most difficult attack to detect and that the attacker can easily destroy any proof by just eating the gelatin mold.

This paper proposed the use of the dynamic property of the skin color. As the finger is pressed on the hard surface of the fingerprint scanner, there occurs change in the color of the skin at the region in contact with the scanner. Such color change is dynamic and occurs only in a live finger. A method to detect and measure the color change is proposed and used to differentiate between a real and fake finger. Section 2 describes the property of the finger color change while Section 3 describes the approaches used to detect and measure the color change. This is followed by Section 4 describing the experimental results before Section 5 concludes the paper.

\section{Finger Color Change}

The main idea of our proposed approach to detect fake finger described in this paper is based on the change in the color of the finger portion in contact with any hard 
surface, such as when the finger is pressed on the finger scanner. As a real live finger is pressed on the hard surface of the scanner, the applied force will cause interaction among the fingernail, bone and tissue of the fingertip. This will alter the hemodynamic state of the finger, resulting in various patterns of blood volume or perfusion [9]. Such pattern is observable at the fingernail bed [9],[10] and at the surrounding skin region in contact with the scanner. The compression of the skin tissue at the contact region with the scanner will cause the color of the region to change to whiter and less reddish compared to the normal portion. This is because blood carries hemoglobin which is red in color. When the finger is pressed onto the hard surface, the amount of blood that can flow to the skin region in contact with the surface is limited due to the force exerted which constricts the capillaries at the fingertip. With less blood flow, the color of the skin region will change to less reddish, resulting in whiter appearance compared to the normal finger. Figure 1 shows the property of the color change before (Fig. 1a and 1c) and after the finger is pressed on a hard surface (Fig. 1b and 1d). For comparison, Figure 2 shows the color of the fake finger made using gelatin before and after the finger is pressed.

As shown in figures 1 and 2, the portion of the finger in contact with the hard surface will show change in color. We postulate this property to be true even for people

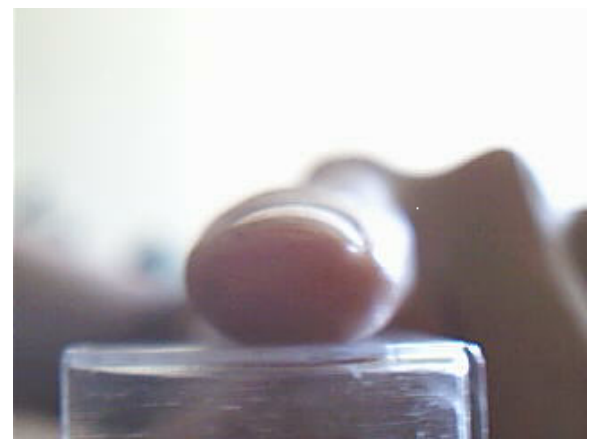

(a) Real finger before pressing

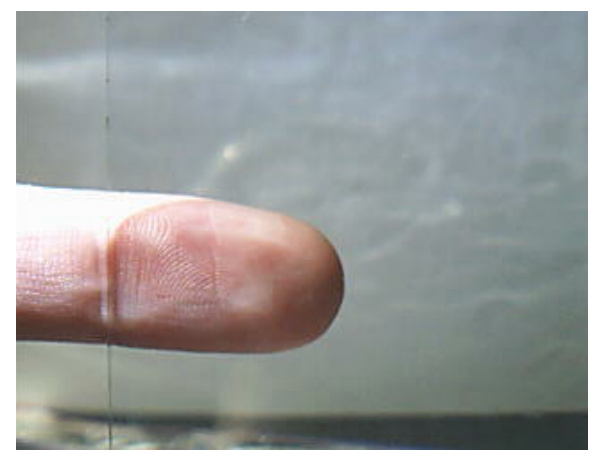

(c) Real finger before pressing

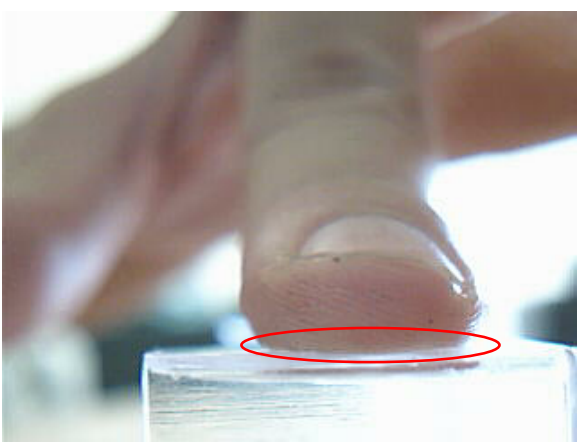

(b) Real finger after pressing

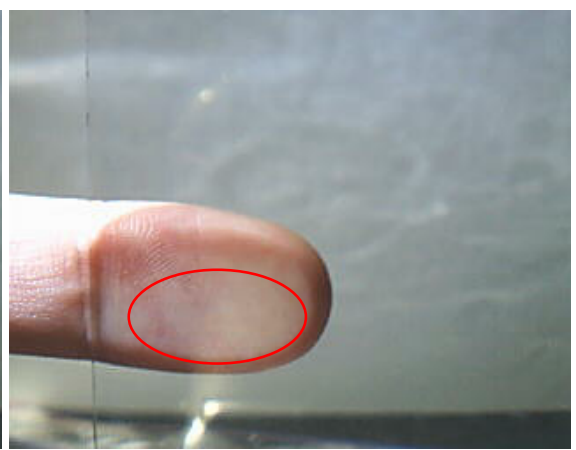

(d) Real finger after pressing

Fig. 1. Images of a real finger before and after pressing on a hard surface 


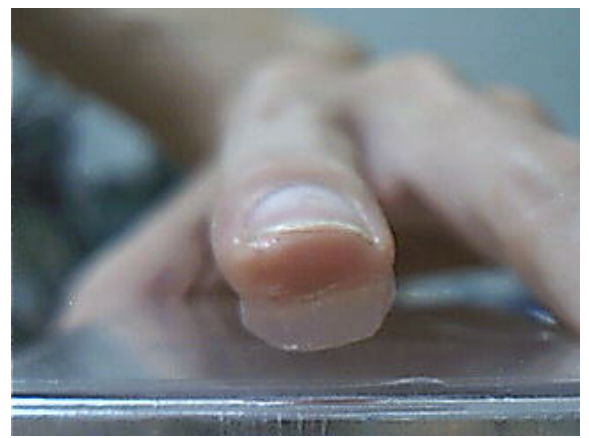

(a) Fake finger before pressing

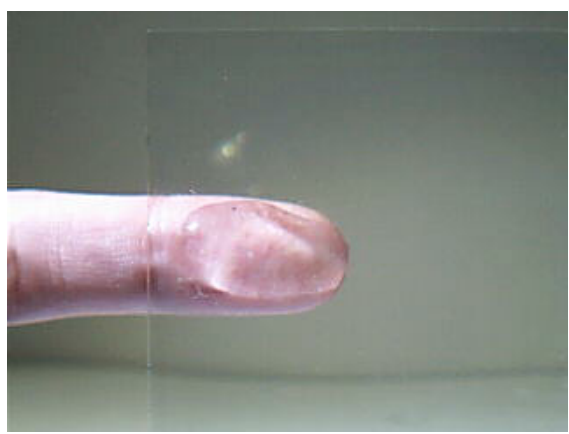

(c) Fake finger before pressing

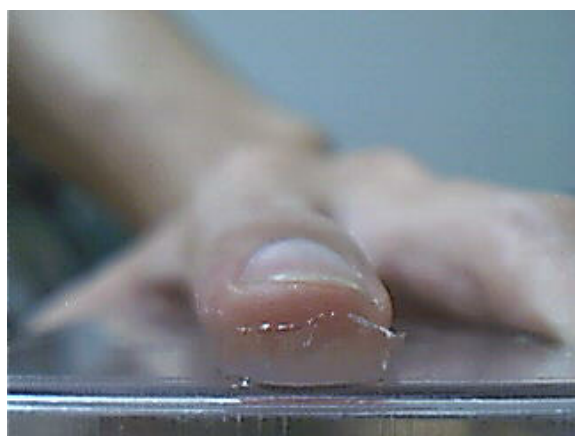

(b) Fake finger after pressing

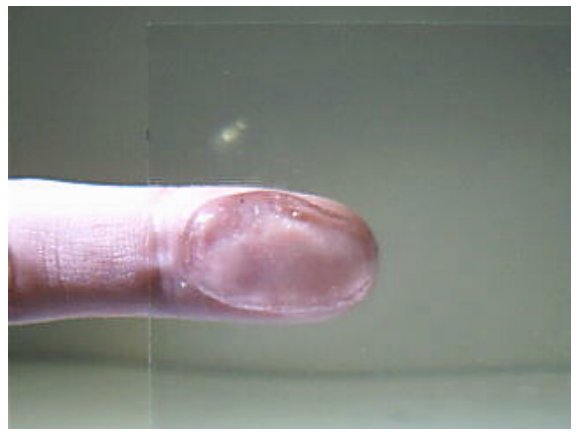

(d) Fake finger after pressing

Fig. 2. Images of a fake finger before and after pressing on a hard surface

of different age, ethnicity or gender and is not sensitive to the type or condition of the skin, such as dry, wet or oily. However, a fake finger will not have such a property as mold will contact the surface first and the user will have to be careful when pressing the soft gelatin mold on the hard surface. As this property is dynamic and repeatable for all live fingers, it can be used to detect the liveness of the finger. The proposed method will only require the use of ordinary low cost digital camera, such as those commonly used in the mobile phones or PCs.

\section{Fake Finger Detection Methodology}

To quantify the change in the color of the finger, we first model the background region using a Gaussian model when the finger is not present. When a substantial change is detected, a finger is present. This image is saved as the initial image, $\mathrm{I}_{\mathrm{I}}$. Then, the images are continuously captured until the finger is pressed on the sensor and then lift off. The finger image with the entire finger pressed on the sensor, which gives the largest contact area, is taken as the desired image, $I_{D}$. Image $I_{I}$ is then aligned to $I_{D}$ based on the tip and the medial axis of the finger as shown in figure 3 . The tip is defined as the point where the medial axis of the fingertip cuts the border of the fingertip. 


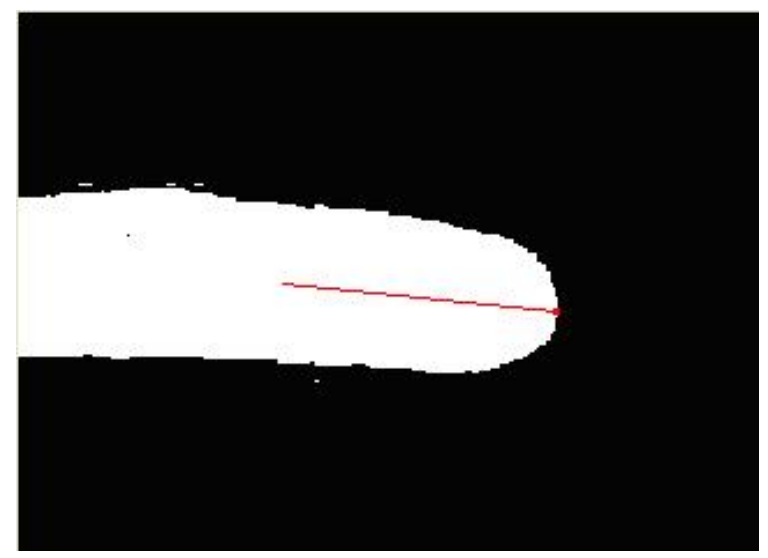

Fig. 3. Alignment of finger using the finger tip. The red line is the medial axis while the red square dot at the boundary between the finger and background is the tip point.

Subsequently, the foreground region of the finger is smoothed using an averaging filter. Then the region is divided into $n_{l} \times n_{2}$ non-overlapping square blocks of size $s$ $\times s$, beginning from the medial axis of the finger. Since we are interested in detecting the color change only, we convert the original image in RGB into CIELa*b* color space which provides good results experimentally.

The color of the fingertip before touching the hard surface is homogeneous. Thus we regard the chrominance component of all the $n_{1} \times n_{2}$ blocks in the initial image $\mathrm{I}_{\mathrm{I}}$ (before pressing) can be modeled using a single Gaussian distribution, $\mu_{o}, \sigma_{o}$. This would be taken as the reference chrominance value for the normal un-pressed fingertip region, $R_{o}$. For each block in the pressed image $\mathrm{I}_{\mathrm{D}}$, clustering using hierarchical $\mathrm{k}$-means [11] is performed on the chrominance component. Then the dominant clusters with homogeneous chrominance value of center $\mu_{l}$ and standard deviation $\sigma_{l}$ different from $\mu_{o}, \sigma_{o}$ found from the $n_{l} \times n_{2}$ blocks is taken as the reference value for the compress region $R_{l}$ of a pressed fingertip. This is then repeated for all the $k$ images of $\mathrm{I}_{I}$ and $\mathrm{I}_{D}$ in the training sets to obtain the overall reference value for the normal $R_{o}\left(\mu_{r o}, \sigma_{r o}\right)$ and pressed region $R_{l}\left(\mu_{r l}, \sigma_{r l}\right)$ of the individual.

Given a pixel $x_{i, j}$, its similarity with respect to the normal $R_{o}$ or pressed region $R_{l}$ can be quantified using the distance measure:

$$
D_{i, j}^{t}\left(x_{i, j} \mid \mu_{r t}, \sigma_{r t}\right)=\sqrt{\frac{\left(x_{i, j}-\mu_{r t}\right)^{2}}{\sigma_{r t}^{2}}} \quad ; t=0,1
$$

The pixel $x_{i, j}$ is assigned to its proper region, $R$, based on the following thresholding operation:

$$
R\left(x_{i, j}\right)=\left\{\begin{array}{cc}
0 & D_{i, j}^{o}<\alpha_{o} \\
1 & D_{i, j}^{1}<\alpha_{1} \\
2 & \text { otherwise }
\end{array}\right.
$$


where $\alpha_{o}, \alpha_{l}$ are the thresholds for the similarity measure to the region $R_{o}$ and $R_{l}$ respectively.

For each block $n$ in the $n_{1} \times n_{2}$ blocks of $I_{I}$ and $I_{D}$, the likelihood that $n$ belongs to the category normal $\left(R_{0}\right)$, pressed $\left(R_{l}\right)$ or otherwise $\left(R_{2}\right)$ can be obtained from the dominant homogeneous region assigned to the pixels in it as:

$$
R\left(n_{i, j}\right)=\bmod \left(\left.R\left(x_{i, j}\right)\right|_{x_{i, j} \in n_{i, j}}\right)
$$

When verifying the liveness of a finger, the category assigned to each of the $n_{1} \times n_{2}$ blocks in images $I_{I}$ and $I_{D}$ is determined using equations (2) and (3). Then the finger is considered real if it satisfies the following criteria:

$$
\left.R\left(\left.n_{i, j}\right|_{I_{I}}\right) \in R_{o} \forall n\right|_{I_{I}} \bigcap \bmod \left(R\left(\left.n_{i, j}\right|_{I_{D}}\right)\right) \notin R_{2} \cap \sum R\left(\left.n_{i, j}\right|_{I_{D}}-\left.n_{i, j}\right|_{I_{I}}\right)>\alpha_{R}
$$

where $\alpha_{R}$ is the threshold for real finger verification.

\section{Experimental Results}

In order to evaluate the proposed approach, a database of images was collected using the prototype setup shown in figure 4.

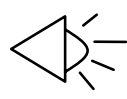

light
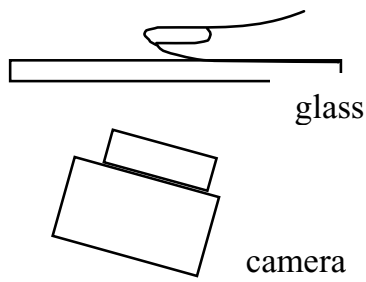

camera

Fig. 4. Prototype system setup for data collection. The camera used is a PC camera with $0.5 \mathrm{M}$ pixel resolution while the light source is obtained from a series of LED.

The images were collected from 25 human subjects using white LED light source. Prior to any capture, the background image without the presence of finger was first captured. Then two images were acquired from each subject, one before the finger pressed the glass and the other after the finger pressed the glass. No guidance was given to the subjects on how they should press their finger except that they were told to press as if they were using a fingerprint recognition system. This was then repeated with the subject wearing a gelatin mold to simulate fake finger. A new gelatin mold was made for each subject since the gelatin mold quality deteriorates with time. Thus we have a total of 50 images (before and after pressing) for real fingers and another 50 images (before and after pressing) for fake fingers in the dataset. All these images were used in the performance evaluation of the proposed system. 
Based on this dataset, we are able to correctly detect all real fingers and achieving $80 \%$ accuracy in detecting the fake finger as fake. Table 1 show the result obtained.

Table 1. Result for real and fake finger detection

\begin{tabular}{|c|c|c|}
\hline & Correct detection rate & False detection rate \\
\hline Real finger & $100 \%$ & $0 \%$ \\
\hline Fake finger & $80 \%$ & $20 \%$ \\
\hline
\end{tabular}

Figure 5 shows some sample results obtained for the real and fake finger respectively. The errors in detecting the fake finger occur when the gelatin mold is made very thin and properly stuck to the real finger before touching the glass surface or due to error in the segmentation process. We found that in some cases, the fake finger has been detected even before pressing it. This is because the mold is not well made with bubbles which will be rejected as the homogeneity constraint of the finger is not valid anymore.

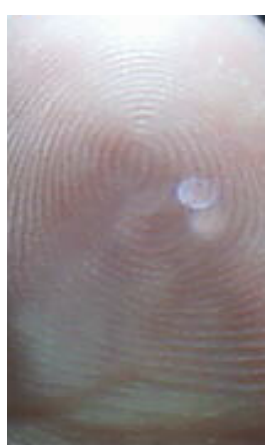

(a) real finger before pressing

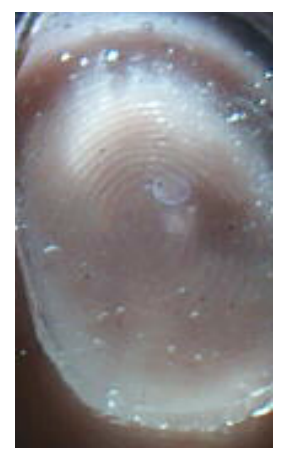

(d) fake finger before pressing

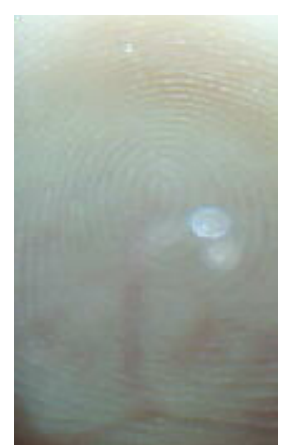

(b) real finger after pressing

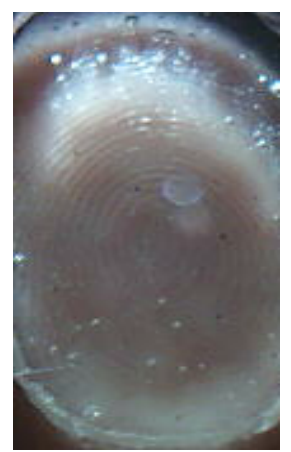

(e) fake finger after pressing

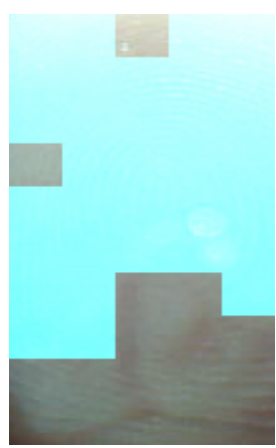

(c) change detected

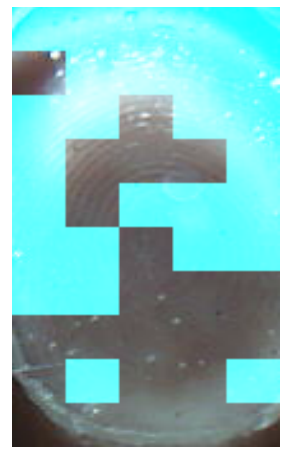

(f) change detected

Fig. 5. Results of color change detection obtained on a real and the corresponding fake finger of the same subject. The colored part is the detected change. 


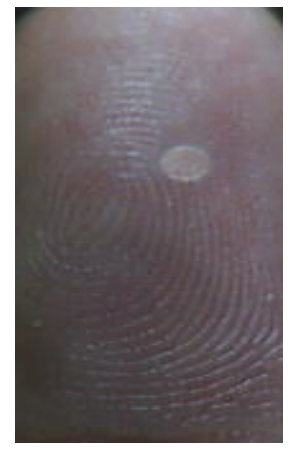

(g) real finger before pressing

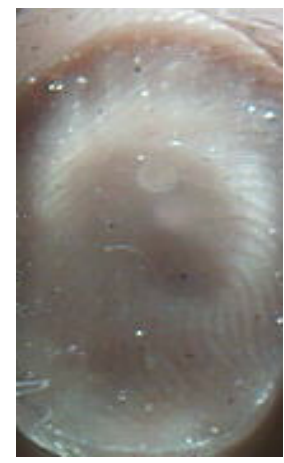

(j) fake finger before pressing

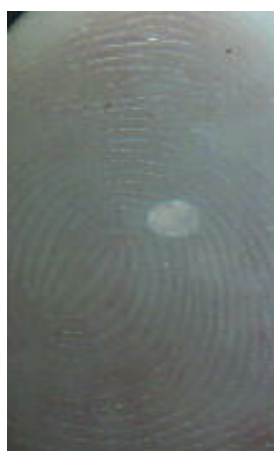

(h) real finger after pressing

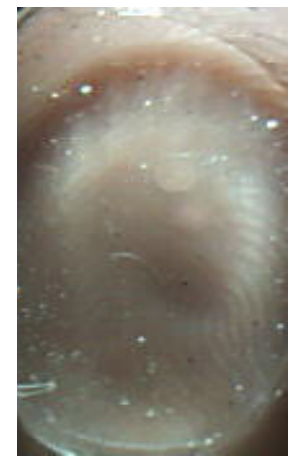

(k) fake finger after pressing

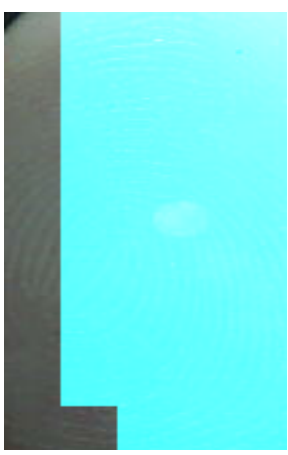

(i) change detected

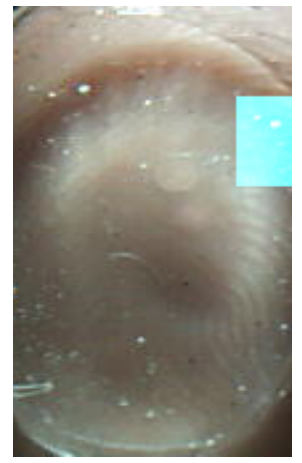

(l) change detected

Fig. 5. (continued)

The advantages of the proposed method are that it is fast since the capture is done real time and does not have to wait for perspiration to set in as in [5]. It also does not require the use of expensive hardware as in [12] and has no implication on the privacy of the user as it does not reveal the medical condition of the person. In addition, it also does not require careful interaction of the user with the scanner as in [6] and thus can be readily deployed for mass users without prior user training.

\section{Conclusion and Future Works}

This paper presented a new approach to detect fake finger based on the property of color change exhibited by a real live finger when the finger touches a hard surface. Such condition arises naturally due to blood perfusion which is universal. The proposed approach is privacy friendly, fast and does not require special action from the user with prior training. The preliminary experimental results indicate that the proposed approach is promising in detecting the fake finger made using gelatin which is particularly hard to detect. 
We are currently working on the use of different light source to improve the accuracy rate of detecting fake finger as well as collecting more data, including those from different ethnic backgrounds, for the purpose of studying the effect of skin color and pressure variation. We recognized that the current setup is only applicable for optical scanner. Thus another future work is to investigate the possibility of using front, back or side view of the fingertip to detect the color change property. These views are useful for non-optical fingerprint scanners where a small digital camera can be installed to capture an appropriate image for detecting the color change property and thus verify the validity of the finger.

\section{References}

1. Ratha, N.K., Connell, J.H., Bolle, R.M.: Enhancing security and privacy in biometricsbased authentication systems. IBM Syst. J. 40(3), 614-634 (2001)

2. Maltoni, D., Maio, M., Jain, A.K., Prabhakar, S.: Handbook of Fingerprint Recognition. Springer, Heidelberg (2003)

3. Matsumoto, T., Matsumoto, H., Yamada, K., Hoshino, S.: Impact of artificial "Gummy" fingers on fingerprint systems. In: Proc. SPIE, vol. 4677, pp. 275-289 (2002)

4. Putte, T., Keuning, J.: Biometrical fingerprint recognition: Don't get your fingers burned. In: Proc. 4th Working Conf. Smart Card Research and Adv. App., pp. 289-303 (2000)

5. Partthasaradhi, S.T.V., Derakhshani, R., Hornak, L.A., Schuckers, S.A.C.: Time-series detection of perspiration as a liveness test in fingerprint devices. IEEE Trans. SMC-Part C 35(3), 335-343 (2005)

6. Antonelli, A., Cappelli, R., Mario, D., Maltoni, D.: Fake finger detection by skin distortion analysis. IEEE Trans. Info. Forensics \& Security 1(3), 360-373 (2006)

7. Schuckers, S.: Spoofing and anti-spoofing measures. Inform. Security Tech. Rep. 7(4), 56-62 (2002)

8. Valencia, V., Horn, C.: Biometric liveness testing. In: Woodward Jr., J.D., Orlans, N.M., Higgins, R.T. (eds.) Biometrics, McGraw Hill, New York (2002)

9. Mascaro, S.A., Asada, H.H.: The common patterns of blood perfusion in the fingernail bed subject to fingertip touch force and finger posture. Haptics-e 4(3), 1-6 (2006)

10. Mascaro, S.A., Asada, H.H.: Understanding of fingernail-bone interaction and fingertip hemodynamics for fingernail sensor design. In: Proc. 10th Int. Symp. Haptic Interfaces for Virtual Environment and Teleoperator Systems, pp. 106-113 (2002)

11. Duda, R.O., Hart, P.E., Stork, D.G.: Pattern Classification. Wiley, Chichester (2001)

12. Nixon, K., et al.: Novel spectroscopy-based technology for biometric and liveness verification. In: Proc. SPIE, vol. 5404, pp. 287-295 (2004) 